\title{
36. THEORY OF STELLAR ATMOSPHERES (THÉORIE DES ATMOSPHÈRES STELLAIRES)
}

\author{
President: A. B. Underhill. \\ Vice-President: R. Cayrel. \\ Organizing Committee: K. H. Böhm, V. V. Ivanov, J. T. Jefferies, R. N. Thomas, S. Ueno.
}

\section{INTRODUCTION}

This report has been prepared by the President with the assistance of the Vice-President, the Members of the Organizing Committee, V. V. Ivanov, P. Conti and of D. G. Hummer. The work of Commission 36 comes in contact with that of many commissions. Some subjects may be dealt with more fully in those reports; some subjects may have been "unlawfully" included here. Considerable choice has been exercised by the President about what to mention and in which section of the report to place it. The final product is only indicative of the large amount of work going on. An extensive bibliography has been compiled and will be distributed to commission members.

Commissions 12, 27, 29, 44 and 45 deal with observational material which our theories attempt to interpret, thus it is essential to study the reports of these commissions to appreciate the diversity of material for which theories are needed and against which theories may be checked. The theoretical treatment of the problems of planetary atmospheres, Commission 16, is not entirely divorced from that of stellar atmospheres. To set realistic inner boundary conditions on our atmosphere models and on our thinking, we must consider the material of Commission 35 while to set the outer boundary conditions we may consider the physical state of planetary nebulae which is one subject of Commission 34. When we wish to apply ideas about the interactions between radiation, atoms, ions and molecules we use material which lies in the province of Commission 14.

In the report period two conferences directly concerned with model atmospheres and the prediction of stellar spectra were held, namely the Third Harvard-Smithsonian Stellar Atmospheres Conference in 1968 on the subject of model atmospheres and predicted spectra based on the hypothesis of LTE, and IAU Colloquium no. 2 in Munich in 1969, on the subject Spectrum Formation in Stars with Steady-State Extended Atmospheres. The proceedings of the former conference contain a homogeneous grid of models with effective temperatures between 4000 and $50000 \mathrm{~K}$ and values of $\log g$ between 2 and 5 . Information about opacities and $\mathrm{H} \gamma$ profiles are also given. The calculations were done by Carbon, Gingerich and Kurucz. This material has been edited by Gingerich and was published by the M.I.T. Press. The proceedings of the Munich Conference have been edited by Wellmann and Groth and have been submitted to Astronomy and Astrophysics as a supplement. Papers on the theory of stellar atmospheres can also be found in colloquia and conferences sponsored by the Commissions mentioned above.

\section{A. THE STUDY OF STELLAR ATMOSPHERES}

\section{Model atmospheres and synthetic spectra}

\section{a. Based on the hypothesis of LTE}

In addition to the work reported at the Third Harvard-Smithsonian Symposium, systematic studies of model atmospheres and synthetic spectra for B stars and for the sun have been conducted by Aller, Ross, Leckrone and others, and for B 6 to O 9 stars by Underhill. Morton and Adams, Bradley, and Hickok have produced line-blanketed models of types B 4 to $\mathrm{O} 5$ from which they 
have derived a scale of effective temperature and bolometric correction for early-type main-sequence stars. The question of whether the absorption-line spectrum which could be predicted matches the spectra observed for the above line-blanketed models has not been studied. Strom and Strom have continued to study the effects of opacity from silicon and the light elements on model atmospheres and have predicted the continuous spectra of late B and A stars. Böhm-Vitense as well as Hunger, Klinglesmith and others have made models for helium-rich stars. Models for white dwarfs have been computed by Matsushima and Terashita, by Kolesov in Leningrad and by Bues in Kiel. Models for A, F and G stars are being made by Peytremann et al. at Geneva. A grid of models for yellow supergiants has been prepared by Parsons while Krishna Swamy as well as F. Praderie have published models and synthetic spectra for F and $G$ stars of various sorts. Cayrel-de Strobel has made sets of scaled solar models to investigate abundance differences in $G$ and $K$ stars. Matsushima has worked on solar type models. A considerable range of spectral type has been surveyed roughly by De Jager and Neven who published models based on an adopted temperature law and calculated synthetic spectra using simplified concepts. Problems connected with late type model atmospheres have been studied by Auman, Bodenheimer, Kandel, Kumar et al., Tsuji, and by Vardya. The analytical representation of models and of synthetic spectra is being studied by Sapar, Kuusik and Viik at Tartu Observatory.

\section{b. When the hypothesis of LTE is not made}

In this case the level populations of the atoms and ions causing the opacity (continuous and line) must be found from the equations of statistical equilibrium and the source function must be modified in an appropriate manner. Mihalas, Stone and Auer have studied early type models when the continuous spectrum due to hydrogen and to helium is not in LTE and when radiative transmission in Lyman, Balmer, Paschen and Brackett $\alpha$ is explicitly considered. It is found that the temperature distribution ceases to decrease rapidly outwards, as in LTE line-blanketed models, and that a small temperature rise may occur. Feautrier has also shown that such a temperature rise may result as $\tau$ becomes very small. Strom, Strom and Kalkofen, Mihalas, and Lambert and Pagel have discussed the observable effects of non-LTE on the shape of the Balmer and Paschen continua of models. It appears that the change in colour and the size of discontinuities are at the limit of detection by present methods of observation. Pagel has considered the effect of atomic collisions with reference to establishing LTE in stellar atmospheres.

\section{Problems in line formation; astrophysically oriented spectroscopic information}

See also Section C. Athay and Skumanich have considered the theoretical interpretation of the Wilson-Bappu effect as well as the effects of non-coherent scattering on the shape of the curve of growth. The question of how to represent the source function in multiplets has been studied by Avrett. The model atmosphere studies often include predictions of the $\mathrm{H} \gamma$ line and sometimes a few other lines. Warner has considered the effects of pressure broadening on solar and stellar curves of growth while Gussmann has indicated how the weight-function method may be cast in a more useful manner. Study of the regimes of quasistatic and of impact Stark broadening of hydrogen has been continued by N. Feautrier, F. Praderie and van Regemorter while Kuusik has discussed the significance of scattering and absorption in the hydrogen lines of stellar spectra. Possible sources of opacity in late type atmospheres and their dependence on temperature and pressure have been investigated by Linsky, Morris and Wyller, and by Vardya, while Fay has studied the $\mathrm{CN}$ molecule in detail.

\section{The spectra of $O$ and $B$ stars; the helium problem}

Temperature scales for A and B stars have been established by Heintze and by Wolff, Kuhi and Hayes as well as by Morton, by comparing measured energy distributions in the continuous spectrum with those predicted by means of model atmospheres. Strom and Peterson have made 
surface-gravity determinations for main-sequence B stars by fitting observed and predicted $\mathrm{H} \gamma$ profiles. Hobbs and C. R. Cowley have used LTE theories to obtain the magnesium abundance in B stars; Snijders has estimated the iron abundance in $\gamma$ Peg and $\zeta$ Cas. The meaning of $O$ type spectra was investigated by Underhill who found that no consistent interpretation could be obtained using LTE theories. Observations of the spectra of halo and globular cluster B stars have been presented by Greenstein, Münch, Sargent and Searle and they have attempted interpretation in terms of $T, P_{e}$ and helium abundance using simple theory. The helium lines are weak relative to other lines in many of these stars although the colours of these stars are like those of B stars. Theoretical studies by $\mathbf{H}$. R. Johnson and Poland, by Hearn and by Shipman and Strom to estimate the importance of non-LTE effects on the HeI line strengths in normal main-sequence stars have yielded contradictory results. Extreme helium poverty and extreme helium enrichment appear to be evident in stellar spectra but spectroscopic analysis is not yet so secure that the helium abundance can surely be determined within a factor two. Underhill has pointed out contradictions in the spectra of B Ia supergiants which may indicate a modest helium enrichment. Hardorp and Scholz have derived the helium abundance in $\tau$ Sco by fitting the wings of profiles and using LTE theory. Baschek and Norris are studying the Her line strengths in B stars.

\section{The spectra of $A$ stars; abundance anomalies}

Many quantitative analyses of the spectra of Ap and Am stars have been made as well as of normal A stars and attempts have been made to determine abundances either by differential curve-of-growth techniques or by LTE model-atmosphere analysis. There is the problem of first finding the correct model ( $T_{\text {eff }}, \log g$ and composition) as well as adopting an appropriate theory of line formation and adequate line broadening mechanisms. Since LTE theories leave no free parameter other than abundance, differing line strengths lead to differing abundances, other factors being equal. Guthrie has summarized much of this work. The variety of results obtained is bewildering and suggests that the methods of analysis used may not be so reliable as the various nvestigators indicate. Unsöld has reviewed the question of the abundance and origin of the elements and concludes that the elements must have been created "en gros". The techniques of abundance determination require a thorough examination with careful consideration of the spectroscopic description of each line, where on the curve of growth it may lie, and whether its intensity is likely to be sensitive to the mode of line formation adopted. Preston has shown that the microturbulence in the Ap stars $\beta \mathrm{CrB}$ and $\gamma$ Equ is small.

\section{The $F, G$ and $K$ stars; the Li/Be problem}

During the report period a considerable number of curve-of-growth, differential curve-of-growth and fine analyses of normal F, G, K stars (main-sequence and supergiants), subdwarfs, cepheids and high-velocity stars has been made and the effects of the lines on the measured colours have been studied. The correction for line blanketing plays an important role in determining where in the HR diagram subdwarfs fall. The helium content of subdwarfs and of population II stars has been deduced by Cayrel and by Strom and Strom from the position of these stars in the HR diagram. Rouse has indicated that the helium content of the solar photosphere may be found from the shape of the wings of strong lines. The abundance analyses for $F, G$ and $K$ stars lead to a variety of results the true significance of which is not entirely clear although it is evident that the value of the not fully understood parameter microturbulent velocity plays an important role. It is generally recognized that the presence of emission components in the CaII resonance lines indicates the presence of a hot chromosphere (see report of IAU Colloquium no. 2). Conti, Danziger and Wallerstein have studied the question of the abundances of lithium and beryllium in stars. Nearly all the observations are consistent with the following statements: (a) a solar-type destruction mechanism (as yet unspecified) operates in G stars and in later types. Its $e$-folding time is a few $10^{8}$ years for $G$ stars and it is more efficient in later type stars. (b) A dilution destruction mechanism 
operates in giant stars. The observations are qualitatively in agreement with predictions by Iben. (c) Pre-main-sequence destruction operates in $\mathrm{K}$ and $\mathrm{M}$ stars. The observations are qualitatively in agreement with predictions by Bodenheimer. (d) The initial $\mathrm{Li}^{7}$ content is roughly constant along the main sequence, at least from 0.5 to 3 solar masses. (e) The initial $\mathrm{Be}$ and $\mathrm{Li}^{6}$ content is lower in $F$ stars than in $G$ stars, or else an additional destruction mechanism operates. This could be an atmospheric destruction by suprathermal protons (Epherre and Reeves). (f) The origin of Li-Be must be spallogenic since stars without any destruction mechanism operating have spallation ratios of $\mathrm{Li}^{6}, \mathrm{Li}^{7}$ and $\mathrm{Be}^{9}$. (g) It is not yet known whether the $\mathrm{Li}-\mathrm{Be}$ content observed in stars is galactogenic or autogenic in origin.

\section{Stars with molecular bands in their spectra}

Analysis of spectra has been attempted in a few cases and models have been made by Vardya and colleagues, Tsuji, and Myerscough. Attention has been directed chiefly at finding the abundance of lithium and of the light elements $\mathrm{C}, \mathrm{O}$ and $\mathrm{N}$ in $\mathrm{M}$ stars as well as in the hydrogen-deficient carbon stars. One section of the book Low Luminosity Stars (Ed. S. S. Kumar) is devoted to the problems of the atmospheres of cool dwarf stars. Polarization and obscuration by particles in the extended atmospheres of late type stars is receiving attention from Dyck and H. R. Johnson and from Krishna Swamy and Wickramasinghe.

\section{Microturbulence and rotation}

The question of microturbulence $v s$. abundance for causing the strengthening of lines in stellar spectra particularly in stars of types $\mathrm{A}$ to $\mathrm{F}$ has been studied by Baschek and Reimers, Barry, Bonsack and Culver, Hardorp and Scholz, McNamara, and Preston and Cathey. Definite evidence from line profiles has been obtained by Hardorp, Bidelman and Prölss that the microturbulent velocity is less than $2 \mathrm{~km} \mathrm{~s}^{-1}$ in $3 \mathrm{Cen} A$ and by Preston that it is less than $3 \mathrm{~km} \mathrm{~s}^{-1}$ in $\beta \mathrm{CrB}$ and $\gamma$ Equ. It is doubtful that spectral peculiarity is connected with microturbulence or that microturbulence is the major factor causing stars of the same F, G type or $(b-v)$ colour, to have differing values of the $m_{1}$ index. The effects of rapid rotation on continuous and line radiation from stars has been investigated by Collins and his colleagues and by Hardorp and Strittmatter. Underhill has done some numerical experiments to demonstrate how the line shapes are deformed when the rotational broadening is of the order of the natural halfwidth of the line. Rapid rotation of a star should give the star a nonspherical form equivalent to a somewhat extended atmosphere in the equatorial plane. This lack of spherical symmetry has been confirmed by the discovery of the intrinsic polarization of Be and shell stars. The geometric effects of the rotation on the line profile produces broad, shallow lines while the extended atmosphere will produce deeper cores. The true interpretation of the observed lines, which are intrinsically strong lines will be reached when theories that take account of the Doppler effect as well as give a physically true representation of line formation in an extended atmosphere are available. The theoretical predictions to date fall short of this goal.

\section{Wolf-Rayet, Be and variable stars}

The problems of Wolf-Rayet stars are reviewed and a bibliography is given in the Proceedings of the Symposium on Wolf-Rayet Stars (Eds. Gebbie and Thomas). The observational material has increased very much over the last years and theoretical models have been stimulated with, as yet, no final choice between them possible on most of the conceptual features. There is a general agreement that the excitation state originates in a mechanical energy source of some kind and that the thermal structure consists of at least an initial outward rise of $T_{e}$ into line-producing regions from the continuum-producing regions. The atmosphere is, thus, chromosphere-like. Castor, Hummer, and van Blerkom are investigating the influence of optical-depth effects on line profiles. An atlas of WR line profiles is in preparation (L. Smith, Kuhi) to be published by JILA-NBS in 1970. Questions of the influence of structural problems of the WR phenomenon center around the 
suggestions of possible binary character and of mass interchange. The aerodynamical, non-LTE, radiation-transfer coupling remains paramount in atmospheric considerations.

Keller and Mutschlecner at Indiana are initiating studies of atmospheres of cepheids from the standpoint of radiative transfer in moving atmospheres for comparison with the flux from static models. This problem has also been studied by Hillendahl. The physical problem is to determine how well static models, based on the flux coming from the pulsating model at each phase, can represent the atmosphere. Hutchings has continued to study mainly geometric models of the expanding envelopes around $\mathrm{OB}$ supergiants while Kogure has attempted models for the shells of Be stars as have Limber and Marlborough.

\section{B. THE SOLAR SPECTRUM}

\section{The continuum spectrum and the inferred solar model}

The temperature distribution in the solar atmosphere has been a topic of active research during the last three years, supported by new observations of the solar continuum from space in the UV and in the IR. At the Bilderberg Conference, the Utrecht Reference Photosphere was superseded by a new model referred to as the Bilderberg Continuum Atmosphere (BCA). This model is largely based on the inversion of limb-darkening laws in various spectral regions. The BCA is characterized by a very flat temperature minimum at about $4600 \mathrm{~K}$ running from $\tau=10^{-2}$ to $10^{-4}$ and is somewhat cooler than the Utrecht Reference Photosphere in deep layers. The BCA at the time of the Conference conflicted only with non-LTE computations of the K line of Can. Later on, new observations in the UV by Parkinson and Reeves and in the IR by Eddy, Léna and McQueen have shown the necessity of having a temperature minimum of about $4300 \mathrm{~K}$, considerably higher $(\simeq 500 \mathrm{~km}$ over the limb) than supposed before. The BCA was revised accordingly by Gingerich and co-authors, and will be published as the "Harvard-Smithsonian Reference Atmosphere" in Solar Physics. It may be concluded that a very substantial advance has been made during the past three years in knowledge of the temperature distribution in the solar photosphere and low chromosphere, as well as in opacity sources in the continuum. Comparison between these improved semi-empirical models and purely theoretical models in radiative equilibrium, corrected for convective transport in deep layers, and taking into account the blanketing effect are mostly still under way (see Subsection 8).

\section{Abundance of the elements in the solar photosphere}

A very important result is that the disagreement between the iron abundance in the photosphere and in the corona has been shown to be spurious and due to an erroneous absolute scale of FeI oscillator strengths. New absolute measurements made by Huber and Tobey, by Martinez, King and Whaling, and by Grasdalen, Huber and Parkinson have shown that oscillator strengths of Fer lines were about 10 times weaker than believed before. As a result of this, the abundance of iron in the photosphere is raised by a factor of ten and brought to the value $\log (\mathrm{Fe})=7.6$ in the usual scale in which $\log (\mathbf{H})=12.00$. The high resolution and low noise observations of the solar spectrum made by Delbouille and Neven at the International Station of the Jungfraujoch have made possible the identification and the measurement of very weak solar lines. A number of forbidden lines of Fen have been identified by J. P. Swings. Grevesse and Swings have shown that these lines lead to an abundance of iron $\log (\mathrm{Fe})=7.5$ in good agreement with the coronal abundance and now also with the new photospheric abundance derived from permitted lines. The abundance of lead has been revised by Grevesse (blue and UV lines), and Peach. Both authors agree on an abundance close to $\log (\mathrm{Pb})=2 \cdot 0$. Ross, Aller and Mohler in a separate study have found about the same result. The abundance of $\mathrm{Li}$ was found to be lower than believed before by Lynds and by Müller. A value $\log (\mathrm{Li})=1.0$ now seems plausible if blends are taken into account. The abundance of beryllium has been investigated by Envold and Hauge, and by Warner. Warner and various co-authors have 
made an extensive study of the abundance of the elements in the solar photosphere and have published this material in the Monthly Notices of the Royal Astronomical Society (seven papers). Grevesse, Lambert and Mallia have contributed widely to the same task (see mainly Solar Physics). Baschek and Holweger have obtained an abundance $\log (C)=8.5$ for carbon from analysis of center-limb variation of atomic $C_{I}$ lines. They found an excitation temperature of $5200 \mathrm{~K}$ from the $\mathrm{CH}\left(A^{2} \Delta-X^{2} \Pi\right)$ band. Müller, Baschek and Holweger have derived an abundance for $\mathrm{O}$ of $\log (\mathrm{O})=8.8$ from the center-limb observation of OI lines. Ross and Aller have shown how the method of synthetic spectra can be used to determine the chromium abundance and Aller in Proceedings of the Astronomical Society of Australia has summarized all recent results on solar abundances.

\section{Non-LTE computations}

The solar atmosphere has been quite naturally chosen as a suitable medium for non-LTE line and continuum computations. Computations of $\mathrm{CaII}$ and $\mathrm{MgII}$ spectra by $\mathrm{S}$. Dumont have led to the conclusion that the theoretical $\mathrm{K}$ line profile could be brought into rough agreement with observed profiles only if the temperature was continuously decreasing outwards to $4200 \mathrm{~K}$ at $450 \mathrm{~km}$ above the limb. A rather similar conclusion was also reached by Linsky and by Athay and Skumanich. It must be recognized that, at the Bilderberg Conference in 1967, non-LTE computations of $\mathrm{Ca}$ II and $\mathrm{MgII}$ were the only evidence in favour of a low temperature minimum in conflict with evidence coming from the level of the UV continuum. New UV and IR measurements strongly favour a value near $4300 \mathrm{~K}$ and it may be the first time, in the tormented history of non-LTE work, that a non-LTE analysis has preceded any other source of information for finding the actual temperature somewhere in the solar atmosphere. This non-LTE result does rely on the fact that the source function of the $\mathrm{K}$ line can be proved to be in LTE, even at $400 \mathrm{~km}$ above the limb. Avrett has shown that difficulties arise in the non-LTE analysis of the D line profiles if the chromospheric rise is placed very much above the limb. Cuny has computed the full spectrum of hydrogen even allowing for non-LTE in the continuum. She has also computed the effect of the far wing of $L \alpha$ on the UV solar continuum (Third Harvard-Smithsonian Conference). Dalgarno has criticized the validity of the formula used by Cuny at very large distances from the core of the line. Feautrier has computed departures from LTE for $\mathrm{H}$ in a theoretical solar photosphere without lines. Jordan as well as Thomas have thoroughly discussed this problem.

\section{Velocity field}

The statistical description of the velocity field in the photosphere is not much clearer than before. There is a trend to admit a lower microturbulence $(\approx 1.0 \mathrm{~km} / \mathrm{s})$ and accordingly a somewhat higher macroturbulence. Parnell and Beckers have found that the one-dimensional power spectrum of the velocity does not fit Kolmogoroff's law.

\section{Inhomogeneities}

Margrave Jr. and Swihart have shown on a two-stream model that if the assumption of pressure equality on horizontal planes is dropped the effect on the photospheric structure is quite significant. Léna has discussed the effect of inhomogeneities on the interpretation of the infrared radiation of the photosphere. Wilson has studied the stratification of temperature fluctuations in the photosphere.

\section{Polarization}

S. Dumont has shown that the polarization at the edge of the disk comes mainly from neutral hydrogen and not from free electrons of the chromosphere. 


\section{Miscellaneous}

Lambert and Mallia have shown that the absolute wavelengths of Fraunhofer lines are accounted for by convective motions and the gravitational redshift of general relativity.

\section{Work under way}

Most of the work under way has been reported at the Pasadena meeting of the A.A.S. on solar astronomy (1969).

\section{a. The continuum spectrum and the solar model}

Mutschlecner and Keller are comparing the new empirical solar models with theoretical models in radiative equilibrium using Strom's treatment of the blanketing. Further work is under way by Gay, Lequeux, Verdet and co-authors in France, and by Mankin and Strong in the U.S.A. to squeeze the infrared data towards a better knowledge of the solar model. The Harvard-Smithsonian group is preparing a new solar model in which non-LTE effects are allowed for in the interpretation of the observational data. There is still a problem in the interpretation of the solar continuum around $1700-1800 \AA$. Peytremann is computing a theoretical model of the solar atmosphere.

\section{b. Abundance of the elements}

Baschek, Garz and co-authors are ready to publish the iron abundance in the photosphere derived from ionized iron lines. The revision of FeI oscillator strengths also affects the absolute scale of FeII oscillator strengths and the abundance of iron derived is $\log (\mathrm{Fe})=7.6$ in agreement with results derived from FeI or from [Ferr].

\section{c. Non-LTE work}

H. R. Johnson is working on the spectrum of OI. The blanketing effect in non-LTE is at present under study at Boulder and at Meudon.

\section{The solar corona and chromosphere}

Abundance estimates from permitted UV and from forbidden coronal lines in the visible have yielded values, especially for the transition group elements, which are substantially higher than those from standard LTE photospheric analysis and considerable dispute about this question has taken place (Athay; Dupree and Goldberg; Jordan and Pottasch). No arguments have been advanced to contradict the results of the coronal forbidden line analyses which support the higher abundance values. See, for instance, analysis of [FexII] 10747 eclipse data by Eddy and Malville and analyses of coronal lines observed in the 1965 and 1966 eclipses (Jefferies, Orrall and Zirker). Delache has suggested that the corona is enriched in heavy metals. Since the suggested mechanism would not apply to the chromosphere, chromospheric abundances have assumed added importance. From analysis of some chromospheric emission-line data Athay has concluded that the chromospheric abundances are in keeping with the GMA photospheric values. However, Pecker and Pottasch have questioned Athay's analysis and they conclude that the data of Dunn et al. indicate chromosphere abundances close to the coronal values.

To use measurements of coronal forbidden line intensities for diagnostic purposes an understanding of the dependence of the excitation of highly ionized species on electron density and temperature is required. Bély and Blaha have studied Fexv while Rozelot has studied FexII. Zirker has studied various coronal ions in several stages of ionization while Chevalier and Lambert have considered FexiI and $\mathrm{Caxv}$. These studies indicate that certain line ratios are especially valuable for inference of electron densities.

Noyes and Kalkofen have used OSOIV observations of the Lyman continuum to construct models of the quiet chromosphere. A two-component model is required to reconcile UV data, brightness 
temperatures at $\mathrm{mm}$ wavelengths and chromospheric electron densities obtained from eclipse observations. Withbroe has analysed OSOrv limb darkening in spectral lines in the 300-1400 region obtaining results consistent with the model of the transition region due to Dupree and Goldberg.

Photoelectric observations of the limb darkening in the visible continuous spectrum obtained at the 1966 eclipse by Weart and Faller have been studied in part by Weart. Further analysis is being done by K. Gebbie and R. N. Thomas. These studies and those by Henze of 1962 eclipse data on the hydrogen lines indicate that the electron density and probably the temperature are higher than given by the BCA model. Thomas has pointed out that this is probably due to omission of the fact that the hydrogen ionization will not be in LTE. Studies of the NaD lines (Athay and Canfield; Finn, Mugglestone and Young) lead to similar conclusions.

Methods for inferring data directly from line spectra and the shortcomings of these methods have been considered by Jefferies and White, de Jager and Neven, and by Wilson and Worrall. Applications to the solar D-lines have been made by Curtis and Jefferies while de Jager and Neven applied their method to the Or triplet at $7700 \AA$. Jefferies and White showed that data on a single line do not allow a generally unique interpretation; Wilson and Worrall concluded that the assumption of frequency independence is at fault. Hearn has pointed out the non-uniqueness of interpretation of the solar $L \alpha$ line profile and he has shown that the Her lines 584 and 537 can only be uniquely interpreted if supplementary data on the Balmer $\alpha$ line is available.

\section{THE THEORY OF RADIATIVE TRANSFER}

\section{Model atmospheres}

Clearly the outstanding technical advance in the present report period has been the computation of fully non-LTE model stellar atmospheres in radiative equilibrium by Auer and Mihalas using the technique of linearization about trial solutions, which they first applied to the problem of temperature correction in LTE radiative models. The full development of this idea appears to make possible the elimination of many problems which have restricted the application of non-LTE theory to highly-artificial models. A brief exposition of this technique has been given by Skumanich. A considerable number of model stellar atmospheres have been computed in which the assumption of LTE is replaced by one of detailed balancing in the resonance lines. Such calculations have been reported by Mihalas; Mihalas and Stone; Feautrier; Strom, Strom and Kalkofen; and Peterson, as well as by others. Further advances have been reported on the problem of computing a radiative equilibrium model atmosphere in LTE by Feautrier, Henyey, Böhm-Vitense, Baerentzen, and Wickramasinghe and Strittmatter.

\section{Non-LTE line formation}

Considerable progress has been made in the theory of non-LTE line formation. Efficient and flexible new computational procedures have been developed by Athay and Skumanich, Rybicki and Hummer, Grant, Grant and Hunt, Kalkofen, and Kurucz. Among the many papers discussing multi-level atoms are those of Avrett; Avrett and Kalkofen; Athay; Avrett, Beebe, Johnson and Cuny; Finn; Finn and Jefferies; Kalkofen; Peterson and Strom; Peterson and Kalkofen; and Beebe, Johnson and Poland. The last two examine the range of validity of the detailed balance assumption for resonance lines entering statistical equilibrium model calculations. All of the above work is based on the assumption of complete redistribution. Line formation by general non-coherent scattering has been studied by Mathis; Hummer; and Weymann and Williams, who have obtained results substantially different from those based on complete redistribution. The effects of noncoherent electron scattering on the line profiles of early type stars have been discussed by Hummer and Mihalas, and by Auer and Mihalas. An extremely valuable discussion of line formation problems 
in expanding atmospheres was given by Rybicki at the Munich Colloquium of Commission 36. Finally, substantial advances have been made by Wilson; Jones and Skumanich; Krook and Rybicki and by Cannon in our ability to solve numerically transfer problems in media with two and three spatial dimensions. Ivanov has considered the problem of the time variation of a Doppler-broadened resonance line. The extensive use of the Monte-Carlo technique has developed for the solution of various transfer problems. Auer, and Avery and House have investigated transfer with general redistribution. Avery, House and Skumanich have examined the transfer of resonance radiation in finite cylinders and House and Cohen have treated polarized radiation in magnetic fields. Magnan has studied line formation in expanding and rotating atmospheres. House and Avery give a review of applications of the Monte-Carlo technique to transfer problems.

\section{Curve-of-growth theory}

Further refinements of the curve-of-growth theory have been reported by McClatchey and by Pecker. Rosendhal has examined the effects of electron scattering and Edmonds has considered the relative effects of micro-turbulence on various forms of curves of growth. Athay and Skumanich have published Milne-Eddington curves of growth for non-coherent scattering. Useful tables of equivalent widths have been given by Golden and a useful approximation by Goldman. Band models have been developed by Yamamoto and Masaru and by Zachor. Golden and Kyle have independently worked out the Doppler analog to the Elsasser band model and Golden has found an expression for the Voigt analog. Van Blerkom and Hummer have solved the non-LTE overlapping line problem using the Golden-Kyle formula.

\section{Miscellaneous}

The vexing problem of a transfer equation for dispersive inhomogeneous media has been discussed by Zheleznyakov and Pomraning. Generalized Eddington approximations have been discussed by Sherman, by Huang and by Pomraning. Pomraning has also given a variational form of the transfer equation. The computation of special functions has received much attention. The efficient computation of exponential integral functions is discussed by Armstrong, and by Cody and Thatcher. The Voigt function is discussed by Armstrong, by Reichel, and by Chiarella and Reichel. Formal radiative transfer and some related computational procedures have also received much attention. However, limitations of space prohibit a discussion of the field. Books of interest that have been published recently are Spectral Line Formation by Jefferies, Linear Transport Theory by Case and Zweifel, Theory of Stellar Spectra by Sobolev and the collection Resonance Lines in Astrophysics, edited by Athay and Mathis.

\section{Work in the Soviet Union}

Research on the theory of stellar atmospheres in the U.S.S.R. centered around radiative transfer problems. Two groups have studied transfer of radiation in line frequencies. These are the Leningrad University group (V. V. Sobolev, D. I. Nagirner, V. V. Ivanov) and the group from the Institute of Atomic Energy, Moscow (Yu. Yu. Abramov, A. M. Dickhne, A. P. Napartovitch). The problem is studied under the usual assumptions of two-level atoms and complete frequency redistribution. The approach is mainly analytical. Both stationary and non-stationary problems have been considered. Attention is paid primarily to the study of the asymptotic properties of the solutions of transfer equations for various standard problems. The usual place for the publication of the results of these groups is the journal Astrophizika. A comprehensive review of the analytical results will be found in the book Radiative Transfer and Spectra of Celestial Bodies by V. V. Ivanov, which is now in press (Nauka Publ. House, Moscow, approx. 400 pp.). Extensive tables of the generalized $\mathrm{H}$ - and related functions for the case of the Voigt profile with the continuous opacity taken into account are being prepared for publication by D. I. Nagirner. The tabulation of the generalized $\mathrm{X}$ - and $\mathrm{Y}$-functions with Doppler profile is now under way in Leningrad. Substantial progress in 
another branch of radiative transfer theory, namely, anisotropic scattering, has been achieved by V. V. Sobolev. In particular, he has found the complete solution of the so-called "pseudo-problems" of anisotropic scattering as formulated by Chandrasekhar some 20 years ago. Quite general results are also obtained in the study of the asymptotic properties of the solutions of transfer equation for anisotropic scattering. Some of these asymptotic results were found independently by H. C. van de Hulst in Leiden. The results of V. V. Sobolev are being published in Astronomiceskij Zurnal and in Astrophizika. Using the ideas of V. A. Ambartzumian, Byurakan astronomers have attacked analytically several model non-linear radiative transfer problems (V. Y. Terebizh et al.).

\section{Problems related to planetary atmospheres}

During the report period much work on multiple light scattering in plane atmospheres has been done by H. C. van de Hulst and his colleagues K. Grossman, R. E. Danielson, D. R. Moore, J W. Hovenier and J. E. Hansen. This work will be summarized in book form by Van de Hulst.

\section{Radiative transfer in extended atmospheres}

Radiative transfer in spherical geometry has been studied by Bellman, Kagiwada, Kalaba and Ueno using the method of invariant imbedding in particle-counting technique. The diffuse transmission of light from a central point source through an inhomogeneous spherical shell as well as diffuse reflection of solar rays by a spherical shell has been studied. Ueno, Kagiwada and Kalaba have derived the functional equations for the scattering and transmission functions and intensity in an inhomogeneous, anisotropically scattering spherical shell atmosphere with radial symmetry allowing for an internal radiation source, an absorbing core and a reflecting boundary. Leong and Sen have obtained some of the same analytical results using the probabilistic method. Uesugi and Tsujita have applied Chandrasekhar's invariance principle as well as the principle of invariant imbedding to some aspects of the problem in cylindrical and spherical media and Matsumoto has looked at time-dependent problems. Although the functional equations for the scattering and transmission functions of the search-light problem in three dimensions have been obtained, it has not yet been possible to solve the complicated integro-differential equations numerically. Radiative transfer in an inhomogeneous infinite cylinder has been studied by Heaslet and Warming and by Buell, Kalaba, Ruspini and Ueno. Multiple Compton scattering in a spherical atmosphere was studied by Code. Aspects of radiative transfer in moving atmospheres have been considered by Kulander, by Rybicki and Hummer, by Magnan and by Bellman, Kalaba and Ueno. The integralequation method has been applied by Kalkofen to this problem. Krook and Rybicki have studied the source function and emergent intensity in a dynamically unstable, three-dimensional planeparallel atmosphere. A similar problem has been studied by Jones and Skumanich. An extension of Sobolev's dynamical treatment of the radiation field in extended atmospheres has been examined by Hirata and Uesugi. The problem of line formation in extended atmospheres including noncoherent scattering and recognizing that LTE is not valid is under study in Japan and at the Munich Observatory as well as elsewhere.

\section{HYDRODYNAMICS AND HYDROMAGNETICS IN STELLAR ATMOSPHERES}

\section{Convection and turbulence in stellar atmospheres}

Progress in the hydrodynamic theory of convection has been slow. In most applications we still have to use the mixing-length theory. However, a few different approaches have been tried: R. Ulrich has applied the concept of a "thermal" (widely used in meteorology) to the convective bubble in the outer convection zone. K. H. Böhrn calculated the approximate amplitudes of the 
different convective modes, assuming that the effects of turbulence can be described by a turbulent "viscosity" and a "conductivity". The basic question of the instability criterion has been re-examined by N. R. Lebovitz and A. Kovetz. P. Souffrin and E. A. Spiegel made the interesting discovery that a system consisting of a convectively unstable and an overlying stable zone can be unstable with respect to the generation of gravity waves. Yamaguchi studied growth rates for an atmosphere with a varying superadiabatic gradient. Ponomarenko reinvestigated the development of turbulence in a conducting fluid. Very useful empirical information about convective modes (in the solar outer convection zone) has been presented by $\mathrm{E}$. Frazier. The mixing-length theory (partly with some minor modifications) has been used in the following studies: $K$. H. Böhm pointed out the importance of outer convection zones in cool white dwarfs and calculated such zones; K. S. Krishna Swamy calculated the onset and the efficiency of convection in normal and in hydrogen deficient stars. $\mathrm{He}$ also compared the onset of convection in $\varepsilon$ Eri (normal metal abundances) and in GMB 1830 (metal deficient). S. Mizuno and M. Nishida showed that a change of the assumed values of parameters in the mixing-length theory can lead to large differences in the structure of the convection zone. K. H. Böhm and E. Stückl studied thermal boundary layer effects in a convection zone. W. Unno has tried to improve the mixing-length theory by taking into account (a) the non-locality of convective flow and (b) non-grey radiative transfer in a convective element.

\section{Shock waves and gas dynamic phenomena including mass loss}

Shock wave phenomena have been studied in order to understand the heating of chromospheres and coronas and atmospheric phenomena in pulsating stars. A recent book useful for astronomers working in this field has been published by Zel'dovich and Raiker (Physics of Shock Waves and High Temperature Hydrodynamic Phenomena, Academic Press, 1967). An interesting survey of the Chisnell-Whitham method for shock wave calculation and its application to stellar atmospheres and envelopes is contained in R. P. Stefanik's thesis. The Brinkley-Kirkwood theory has been applied by P. L. Sachdev. Radiation phenomena in shock waves have been investigated by C. J. Bartlett, A. J. Skalafuris and V. V. Zaitsev. Line profiles emitted by acoustic waves have been computed by G. Eriksen and P. Maltby. Considerable effort has been devoted to the study of the hydrodynamic heating of stellar chromospheres and coronas (P. Ulmschneider, R. F. Stein, R. A. Kopp, M. Kuperus, R. Mäckle). Ono, Ischizuke and Taira generalized the Chisnell method so that the propagation of any shock can be treated in arbitrary layers. The problem of conductive heating of the solar chromosphere-corona transition region in a magnetic field and its dynamical consequences has been studied by M. Kuperus and R. G. Athay and by R. A. Kopp and M. Kuperus. The problem of mass loss from stars continues to be of great interest. Using energy considerations and estimating efficiences for different mass-loss processes, I. P. Williams has derived upper limits for the mass loss. The mass loss from OB supergiants has been determined by D. C. Morton. The theory of this effect was discussed by Lucy and Solomon. T. Schmid-Burgk made a detailed numerical study of the mass loss of central stars of planetary nebulae (due to radiation pressure effects). G. S. Bisnovatyi-Kogan and Ya. B. Zel'dovich studied mass loss induced by high atmospheric opacity. The connection between mass loss from stellar coronae and stellar rotation has been investigated by $\mathrm{K}$. Nariai.

\section{Turbulence and convection in hydromagnetics}

The fundamental and difficult problem of the dynamics of a magnetic field in a turbulent medium has been reinvestigated by S. I. Vainshtein and E. N. Parker. A stellar atmosphere problem related to this question is the accumulation of magnetic field lines at the boundaries of convection cells (A. Getling and R. W. Milkey). Overstability and oscillatory convection in the presence of a strong magnetic field were studied by S. I. Syrovatskii and Yu. D. Zhugzhda and by M. Saito and S. Kato. The first two authors discovered an instability with respect to a magnetosonic type of oscillation. 
4. Hydrodynamic and hydromagnetic noise in stellar atmospheres; hydromagnetic waves

Most of the work in this field is stimulated by the hope to understand the heating of chromospheres and coronas and the observed oscillations in the solar photosphere. Very useful new empirical information about the latter problem has been obtained by E. Frazier. Studies of the generation of waves (acoustic, gravity or magnetohydrodynamic) have been made by S. Kato, B. D. Savage, M. S. Howe, J. E. Pfowes Williams. Problems of wave propagation in an atmosphere have been treated by R. F. Stein, A. Getling, M. A. Gintsburg. In the last paper large amplitude waves in a two-fluid model are considered. The response of an atmosphere to gravitational-hydrodynamic waves has been studied by $Y$. Uchida. P. Ulmschneider suggested that the 300 seconds oscillation may be understood as standing gravity waves (Väisälä mechanism). Uchida has explained the Moreton wave emitted by flares as the MHD fast mode propagated through the corona and refracted back to the chromosphere. S. P. Tarafdar developed a magneto-gas-dynamic model of a stellar corona. Acceleration of electrons in a collisionless shock was studied by C. Lacombe and A. Mangeney.

\section{Hydrodynamics in atmospheres of rotating stars}

It was stated by E. Spiegel during the Nice Symposium that Rossby waves might be of importance in the sun and in other stars. This subject has been studied by S. Kato and at present is being investigated by R. Sakurai and P. A. Gilman.

\section{Miscellaneous problems in hydromagnetics}

H. S. Yun has generalized the magnetohydrostatic sunspot models by Deinzer by including the pressure due to the curvature of the magnetic field as part of the surface boundary condition. In another study an azimuthal component of the magnetic field was taken into account. A. Unsöld and G. Godoli have discussed magnetohydrodynamic problems arising in connection with the study of stellar and solar activity.

A. B. UNDERHILL President of the Commission 